\section{High-density mapping of single-molecule trajectories with photoactivated localization microscopy}

\author{
Suliana Manley ${ }^{1}$, Jennifer M Gillette ${ }^{1}$, \\ George H Patterson ${ }^{1}$, Hari Shroff ${ }^{2}$, Harald F Hess ${ }^{2}$, \\ Eric Betzig ${ }^{2} \&$ Jennifer Lippincott-Schwartz ${ }^{1}$
}

\begin{abstract}
We combined photoactivated localization microscopy (PALM) with live-cell single-particle tracking to create a new method termed sptPALM. We created spatially resolved maps of single-molecule motions by imaging the membrane proteins Gag and VSVG, and obtained several orders of magnitude more trajectories per cell than traditional single-particle tracking enables. By probing distinct subsets of molecules, sptPALM can provide insight into the origins of spatial and temporal heterogeneities in membranes.
\end{abstract}

Cell membranes are characteristically heterogeneous both structurally and dynamically. By performing single-particle tracking in live cells, one can access information on the heterogeneities in the motions of individual proteins to provide insights into many cellular events $^{1-3}$. For single-molecule detection, proteins of interest are conjugated to probes such as gold beads ${ }^{4}$ or fluorescentprotein chimeras ${ }^{3}$. However, the number and density of single molecules that can be tracked in an individual cell is limited in traditional single-particle tracking studies because only a single ensemble of molecules is labeled, and particle localization requires that molecular separations be greater than their diffraction-limited size. Thus, single-particle tracking has been unable to probe cell membranes in the spatially and temporally resolved fashion required to determine structural signatures of dynamic heterogeneities.

Super-resolution techniques such as $\mathrm{PALM}^{5}$ enable the imaging of fluorescent-protein chimeras to reveal the organization of genetically expressed proteins on the nanoscale with a density of molecules high enough to provide structural context. Recently, the use of photoactivatable markers for subdiffraction localization has been applied to imaging in living cells, but the dynamics of individual molecules were not resolved ${ }^{6}$. Here we combined the techniques of PALM and single-particle tracking to resolve the dynamics of individual molecules by tracking them in live cells; a method we call sptPALM. We obtained information on the positions of single molecules by activating, localizing and bleaching many subsets of photoactivatable fluorescent-protein chimeras (Supplementary Video 1 online). To enable sptPALM on live cells, we optimized the data acquisition rate and cell viability by exciting the molecules at high intensities, choosing a robust cell line (COS7), operating in a total internal reflection geometry and using a high numerical aperture objective (Olympus APO100XO-HR-SP; 1.65 NA). In addition, we used proteins tagged with $\mathrm{EosFP}^{7}$, a photoconvertible protein with a high photon count and a high contrast ratio between converted and unconverted forms. Using these parameters, we imaged membrane proteins at a rate of 20 frames per second without inducing detrimental effects to living cells (Supplementary Fig. 1 and Supplementary Video 2 online). This permitted the construction of maps of single molecule diffusion for up to thousands of molecules in the plasma membrane, providing a means of obtaining spatially resolved information on cellular dynamics and local environments on the molecular scale.

We transfected COS-7 cells with either of two membrane proteins known to have distinctly different spatial distributions and mobilities: the tsO45 vesicular stomatitis virus $\mathrm{G}$ protein (VSVG) and the human immunodeficiency virus type 1 (HIV-1) structural protein Gag. VSVG is distributed relatively homogeneously across the plasma membrane, where a large fraction freely diffuses $^{8}$; in contrast, Gag multimerizes into immobile virus-like particles (VLPs) that can bud from the plasma membrane ${ }^{9}$. Timeintegrated PALM images (Fig. 1a) reflect the distribution of all molecular peaks localized to $<25 \mathrm{~nm}$. As expected, Gag tagged with tandem dimer EosFP (Gag-Eos) formed bright puncta of 100-200 nm size (Fig. 1b), consistent with electron microscopy data of VLPs. In addition, several larger bright regions (possibly corresponding to clusters of VLPs) and areas of lower than average density (voids) were visible ${ }^{5}$. Unlike Gag-Eos, VSVG tagged with dimeric EosFP (VSVG-Eos) displayed gradual variations in molecular density instead of bright puncta or large heavily enriched domains (Fig. 1b).

In addition to examining the spatial distribution of proteins, use of sptPALM provided dynamic information on individual molecules. As in traditional single-particle tracking, we created trajectories by linking molecular peaks in consecutive frames according to their proximity (Supplementary Methods, Supplementary Figs. 2 and 3 online). We observed mobile and immobile molecules for both Gag-Eos and VSVG-Eos (see example trajectories in Fig. 1c). Unlike traditional single-particle tracking in which a single ensemble of molecules is imaged and tracked until they are

\footnotetext{
${ }^{1}$ National Institute of Child Health and Human Development, National Institutes of Health, Bethesda, Maryland 20892, USA. ${ }^{2}$ Howard Hughes Medical Institute, Janelia Farm Research Campus, Ashburn, Virginia 20147, USA. Correspondence should be addressed to J.L.-S. (jlippin@helix.nih.gov).

RECEIVED 25 OCTOBER 2007; ACCEPTED 19 DECEMBER 2007; PUBLISHED ONLINE 13 JANUARY 2008; DOI:10.1038/NMETH.1176
} 


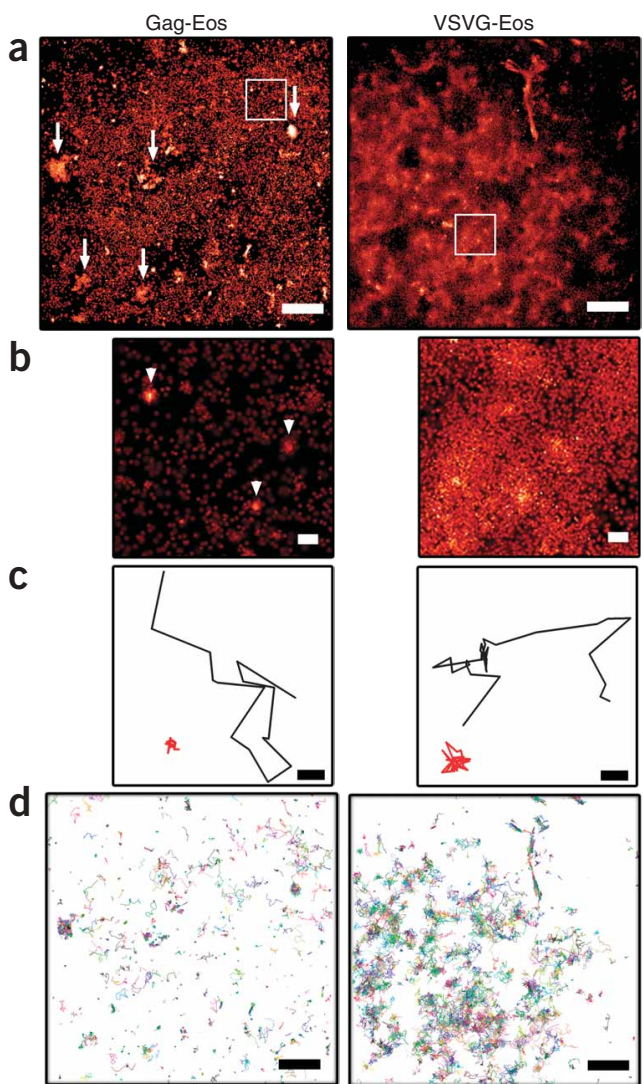

bleached $^{3}$, photoactivatable fluorophores enabled multiple ensembles of molecules to be activated, imaged and bleached. This allowed us to obtain high-density single-molecule trajectories, up to $\sim 50$ per $\mu \mathrm{m}^{2}$ (Fig. 1d). Gag-Eos trajectories varied from apparently immobile populations to mobile populations with pathlengths up to $\sim 1 \mu \mathrm{m}$. In contrast, VSVG-Eos appeared highly mobile, exploring large regions of the plasma membrane. The use
Figure 1 | sptPALM imaging of Gag and VSVG expressed in live COS7 cells. (a) PALM images of Gag-Eos and VSVG-Eos, integrated over $500 \mathrm{~s}(10,000$ images). Molecules are rendered as Gaussian normalized peaks, with a width corresponding to the uncertainty in their position. Arrows indicate large Gag-enriched regions. Scale bars, $2 \mu \mathrm{m}$. (b) Magnified regions, corresponding to boxed regions in a. Arrowheads indicate enriched puncta. Scale bars, $200 \mathrm{~nm}$. (c) Two representative magnified Gag and VSVG single molecule trajectories from the cells shown in a. The tracks represent diffusive (black) and confined (red) movement. Scale bar, $100 \mathrm{~nm}$. (d) Complete sptPALM trajectories of localized Gag and VSVG molecules that are longer than 15 frames (750 ms). Each color indicates a different track. Scale bar, $2 \mu \mathrm{m}$.

of sptPALM allowed localization and tracking of many overlapping trajectories because the distance between fluorescent molecules at any time was greater than several times the width of their point spread function.

A notable improvement afforded by sptPALM is the ability to probe the dynamics of many molecules in a single cell. By comparison, 10-100 cells are typically used to obtain statistically significant single-particle tracking information on individual proteins tagged with conventional labels because each cell yields less than 10 tracks on average ${ }^{10-12}$. We achieved similar statistics with sptPALM on a single cell (Fig. 2a); moreover, the large number of molecular trajectories we obtained allowed us to define molecular environments within individual cells. We calculated the mean-squared displacement (MSD) as a function of time lag, $\Delta \tau$, for trajectories longer than 15 frames $(\sim 3 \%$ of all molecules). For diffusive behavior, we expected the MSD to increase linearly with $\Delta \tau$ for both VSVG and Gag (Fig. 2b). To determine the short-time diffusion coefficients, $D$, for these longer-lived molecules, we used the relationship $\mathrm{MSD}=\mathrm{C}+4 D \Delta \tau$, where $\mathrm{C}$ is a constant offset. A substantial fraction of the trajectories resulted in MSDs that increase in a sublinear fashion (Fig. 2c). This behavior is known as anomalous diffusion: it has been demonstrated for several membrane proteins and lipids $^{13}$, and is hypothesized to be a result of local barriers to diffusion.
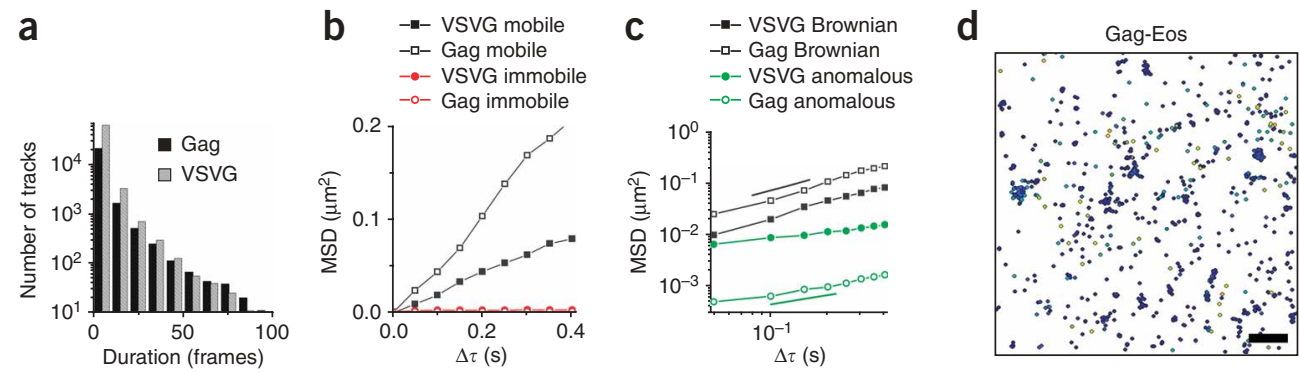

Figure 2 | Analysis of single molecule trajectories in live COS7 cells. (a) Distribution of trajectory durations of Gag-Eos and VSVG-Eos single molecules from the images in Figure 1. The histogram represents all the trajectories detected in a $17 \times 17 \mu \mathrm{m}$ field of the cell membrane with 10,000 images and 50-ms exposures. (b) Calculated MSD for the two Gag and VSVG trajectories shown in Figure 1c. The tracks represent trajectories with diffusive and confined movement. (c) Calculated MSD for Gag and VSVG trajectories that display Brownian diffusion and anomalous diffusion. Short lines are drawn to guide the eye, with slopes 1 (black) and 0.6 (green). (d) Diffusion maps of the Gag- and VSVG-expressing cells illustrated in Figure 1. Each point represents the starting position of one trajectory with a minimum length of 15 frames (750 ms). Molecules with $D<0.01 \mu \mathrm{m}^{2} / \mathrm{s}$ are plotted as dark blue points, while the most mobile molecules are plotted as red points. Representative errors for diffusion coefficients are indicated left of the color bar for the shortest (maximum error) and longest (minimum error) tracks. Scale bars, $2 \mu \mathrm{m}$. (e) Histogram of the distribution of diffusion coefficients of single Gag and VSVG molecules with a minimum track length of 15 frames (750 ms). For fixed VSVG (4\% paraformaldehyde), the distribution of diffusion coefficients was obtained from two cells. For live Gag and VSVG, the distribution of diffusion coefficients was obtained from three cells each. 


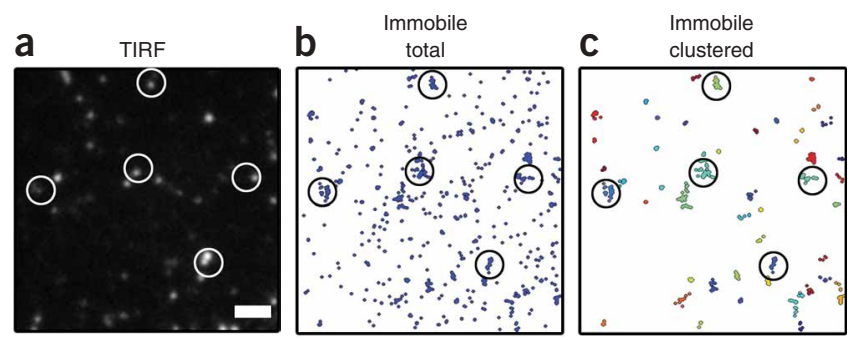

Figure 3 | Cluster analysis on the immobile fraction of Gag. (a) Total internal reflection fluorescence microscopy image of the unconverted Gag-Eos taken under $488 \mathrm{~nm}$ excitation before photoconversion and data collection for sptPALM. Circles indicate corresponding regions in each image. (b) A map of the positions of molecules with $D<0.05$. Each point represents the starting position of one trajectory with a minimum length of 15 frames $(750 \mathrm{~ms})$. (c) Immobile Gag molecules within a $300 \mathrm{~nm}$ radius were grouped as clusters. Each color indicates a separate cluster of at least five molecules. Scale bar, $2 \mu \mathrm{m}$.

The high density of dynamic information available from sptPALM allowed us to create a spatially resolved map of single molecule diffusion coefficients (Fig. 2d) beyond the error owing to finite run length (Supplementary Methods). This map emphasized the large clusters of immobile Gag-Eos molecules, which may correspond to regions where VLPs were concentrated. Notably, although VSVG-Eos is characterized as a highly mobile molecule, we detected less mobile regions that may represent filipodial structures where molecular mobility was slowed by folds in the membrane.

These diffusion maps of VSVG-Eos and Gag-Eos reflect the dynamics of molecules in single cells. By combining data from three cells, we constructed a histogram of diffusion coefficients (Fig. 2e), which confirmed that a larger fraction of VSVG-Eos molecules were mobile than of Gag-Eos. A control experiment on fixed cells transfected with VSVG-Eos revealed greater than $95 \%$ of these molecules had $D<2.5 \times 10^{-2} \mu^{2} / \mathrm{s}$ (Supplementary Fig. 4 online). The average diffusion coefficient measured for the mobile fraction in live cells for Gag-Eos was $0.11 \pm 0.08 \mu \mathrm{m}^{2} / \mathrm{s}$, and for VSVG-Eos it was $0.14 \pm 0.11 \mu \mathrm{m}^{2} / \mathrm{s}$, in agreement with fluorescence recovery after photobleaching (FRAP) ${ }^{8}$ measurements. Diffusion maps such as these reveal dynamic heterogeneities in cell membranes unlike diffusion measurements obtained by traditional single-particle tracking or ensemble measurements such as FRAP. The heterogeneities, in turn, can be characterized further by their size, morphology and number of molecules.

The immobile fraction of Gag may represent molecules trapped in VLPs, which are known to cluster in plasma membrane domains enriched in tetraspanins ${ }^{14}$. These domains appeared as large bright spots when imaged with diffraction-limited fluorescence microscopy (Fig. 3a). We used sptPALM to identify and map the immobile fraction of Gag-Eos (Fig. 3b), revealing structures at scales larger than the dimensions of individual VLPs. To further characterize these structures, we performed a clustering analysis by identifying molecules with neighbors within a $300-\mathrm{nm}$ radius, then grouped molecules with shared neighbors into the same cluster. We displayed all clusters containing a minimum of 5 molecules, which demonstrated that diffraction-limited regions of similar size and intensity may contain dramatically different numbers of molecules (Fig. 3c). Cluster analysis from sptPALM revealed these differences and provided quantitative information inaccessible by total internal reflection fluorescence microscopy. We found that in this cell, 1,074 molecules, representing $67 \%$ of all trajectories shown in the diffusion maps, belong to 54 clusters. Although this information by itself does not allow us to distinguish between different scenarios for cluster formation, a future study of the characteristics of Gag-Eos dynamics in clustered regions could provide insight by revealing whether molecules exhibit reduced mobility or directed motion as a result of interactions with cellular factors that aid VLP budding.

The analyses applied here are only the beginning for this new method; data can be further mined to explore different subsets of molecules based on their spatial organization or dynamics. Although development of probes and instrumentation will certainly lead to improvements in these techniques, presently available reagents and imaging tools are capable of providing new information, as demonstrated in this work. sptPALM can be used to reveal spatially resolved information about membrane protein dynamics. This provides a local context for individual molecules, an important clue for understanding the mechanisms that drive behaviors including clustering and anomalous diffusion, both phenomena ubiquitous to membrane proteins.

Note: Supplementary information is available on the Nature Methods website.

\section{ACKNOWLEDGMENTS}

This project was supported by the Intramural Research Program of the US National Institute of Child Health and Human Development, National Institutes of Health, and performed while S.M. held a National Research Council Research Associateship Award at the National Institutes of Health. We thank D. Blair and A.D. Douglass for providing MATLAB code and helpful discussions.

\section{Published online at http://www.nature.com/naturemethods/ Reprints and permissions information is available online at http://npg.nature.com/reprintsandpermissions}

1. Teramura, Y. et al. EMBO J. 25, 4215-4222 (2006).

2. Yildiz, A. et al. Science 300, 2061-2065 (2003).

3. Douglass, A.D. \& Vale, R.D. Cell 121, 937-950 (2005).

4. Kusumi, A., Sako, Y. \& Yamamoto, M. Biophys. J. 65, 2021-2040 (1993).

5. Betzig, E. et al. Science 313, 1642-1645 (2006).

6. Hess, S.T. et al. Proc. Natl. Acad. Sci. USA 104, 17370-17375 (2007).

7. Wiedenmann, J. et al. Proc. Natl. Acad. Sci. USA 101, 15905-15910 (2004).

8. Kenworthy, A.K. et al. J. Cell Biol. 165, 735-746 (2004).

9. Jouvenet, N. et al. PLoS Biol. 4, 2296-2310 (2006).

10. Wieser, S., Moertelmaier, M., Fuertbauer, E., Stockinger, H. \& Schutz, G.J. Biophys. J. 92, 3719-3728 (2007).

11. Vrljic, M., Nishimura, S.Y., Brasselet, S., Moerner, W.E. \& McConnell, H.M. Biophys. J. 83, 2681-2692 (2002).

12. Ewers, H. et al. Proc. Natl. Acad. Sci. USA 102, 15110-15115 (2005).

13. Feder, T.J., Brust-Mascher, I., Slattery, J.P., Baird, B. \& Webb, W.W. Biophys. J. 70 2767-2773 (1996).

14. Nydegger, S., Khurana, S., Krementsov, D.N., Foti, M. \& Thali, M. J. Cell Biol. 173, 795-807 (2006). 